\title{
Observation of sonified movements engages a basal ganglia frontocortical network
}

\author{
Gerd Schmitz ${ }^{1}$, Bahram Mohammadi ${ }^{2,3}$, Anke Hammer $^{4}$, Marcus Heldmann ${ }^{2}$, Amir Samii ${ }^{3}$, Thomas F Münte ${ }^{2 *}$ \\ and Alfred $O$ Effenberg
}

\begin{abstract}
Background: Producing sounds by a musical instrument can lead to audiomotor coupling, i.e. the joint activation of the auditory and motor system, even when only one modality is probed. The sonification of otherwise mute movements by sounds based on kinematic parameters of the movement has been shown to improve motor performance and perception of movements.

Results: Here we demonstrate in a group of healthy young non-athletes that congruently (sounds match visual movement kinematics) vs. incongruently (no match) sonified breaststroke movements of a human avatar lead to better perceptual judgement of small differences in movement velocity. Moreover, functional magnetic resonance imaging revealed enhanced activity in superior and medial posterior temporal regions including the superior temporal sulcus, known as an important multisensory integration site, as well as the insula bilaterally and the precentral gyrus on the right side. Functional connectivity analysis revealed pronounced connectivity of the STS with the basal ganglia and thalamus as well as frontal motor regions for the congruent stimuli. This was not seen to the same extent for the incongruent stimuli.
\end{abstract}

Conclusions: We conclude that sonification of movements amplifies the activity of the human action observation system including subcortical structures of the motor loop. Sonification may thus be an important method to enhance training and therapy effects in sports science and neurological rehabilitation.

\section{Background}

In 1949, the famous Canadian neuroscientist Donald Hebb coined the phrase "Neurons that fire together wire together", also known as Hebb's axiom, implying that all aspects of an experience give rise to an amalgamated pattern of neural activity, which, if repeated, becomes entrained and more easily elicited.

A case in point of such integrated neural activity shaped by excessive and repeated experience has been auditory-motor coupling in the musician's brain. Musicians create intricate sound-patterns by the movement of their hands. Sounds and movements are thus tightly coupled. Indeed, Haueisen and Knösche [1], using magnetoencephalography, showed that pianists who merely listened to pieces of well-trained piano music showed activation of the contralateral motor cortex. Similar observations have been made by a number of other

\footnotetext{
* Correspondence: Thomas.muente@neuro.uni-luebeck.de

${ }^{2}$ Department of Neurology, University of Lübeck, Lübeck, Germany

Full list of author information is available at the end of the article
}

researchers [2-7]. An important study by Bangert and co-workers compared professional pianists and nonmusicians as they either listened to trained music or performed a short piece of music on a muted piano keyboard while lying in a scanner. The network recruited by professional musicians for listening to music as well as for performing musical actions was highly similar, suggesting transmodal co-activation. This network was speculated to have properties of a transmodal mirror neuron system [7]. Another example of coupling between motor and auditory brain areas has been reported by Lotze and co-workers [2] who compared fMRI activations of professional and amateur violinists during actual and imagined performance of a violin concerto. Besides activations in motor areas, professionals exhibited higher activity of the right primary auditory cortex during silent execution indicating increased audio-motor associative connectivity. Motor and auditory systems were coactivated in this study and co-activation was modulated as a function of musical training. To pinpoint the areas

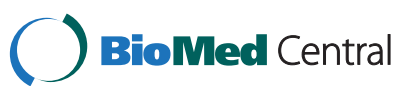


involved in audiomotor coupling Baumann et al. [5] investigated skilled pianists and non-musicians during silent piano performance and motionless listening to piano sound. A network of secondary and higher order auditory and motor areas was observed for both conditions among which the lateral dorsal premotor cortex and the pre-supplementary motor cortex (preSMA) played a significant role. While the majority of studies on audiomotor coupling has employed musical stimuli, Baumann and Greenlee [4] investigated real-life moving objects characterized by multisensory information. Random dot patterns moving in phase, moving out-of -phase, or being stationary were accompanied by auditory noise moving in phase, moving out-of-phase, or not moving. When the sound source was in phase with the visual coherent dot motion, performance of the participants was best. FMRI showed that auditory motion activated (among other regions) the superior temporal gyrus (STG) on the right more than on the left. Combined audiovisual motion activated the STG, the supramarginal gyrus, the superior parietal lobule, and the cerebellum.

One function of such integrated networks might be the facilitation of movement patterns. This notion has triggered interest, for example in the fields of sports science [8] or neurorehabilitation [9-11], to induce audiomotor coupling to enhance movement (re)-acquisition. The sonification of human movement patterns represents an approach to enrich movements - that are not normally associated with typical sound patterns - by adding an auditory component to the movement cycle $[12,13]$. This is achieved by transforming kinematic as well as dynamic movement parameters into sound. Emerging sound patterns are typical for a certain movement pattern. The additional movement acoustics can be exploited by multisensory integrative brain areas [8] and the transmodal mirror neuron system [7] which then might lead to a more stable and accurate representation of the movement. Congruent audiovisual motion information results in more accurate percepts, increased motor performance as well as enhanced motor learning. Behavioral benefits have been reviewed by Shams and Seitz $[14,15]$ who argue that a larger set of processing structures is activated by multimodal stimuli. Moreover, Lahav et al. (2007) hypothesized an audiovisual mirror neuron system with premotor areas inherently involved and serving as an "action listening" and "hearing-doing mirror neuron system", with the latter being dependent on the individual's motor repertoire.

In learning new skills in sports or relearning basic skills in motor rehabilitation the observation of the skill and its reproduction are key elements. Observational motor learning can be achieved by visual perception, but vision is not the only sense providing information about movement patterns: especially in the temporal domain auditory perception is much more precise than visual perception. Unlike the movements of the pianist on the piano-keyboard, movements associated with running, swimming, or walking only give rise to little if any auditory information mostly limited to short movement phases, for example when the shoe hits the ground or the racket hits the ball. Even auxiliary auditory information provided by trainers or therapists is reduced to brief accents, such as clapping with the hands or the use of a drum. Previous research has indicated that continuous and more complex forms of auditory movement information like Audification or Sonification of naturally mute phases of movements can efficiently improve motor performance, e.g. when sonifying the inner hand pressure in freestyle swimming [16].

In the present study we first demonstrate that a movement sonification of breaststroke based on kinematic parameters leads to more precise judgements of swimming velocity differences when combined with a video of a breaststroke avatar. Second, to study the neural substrate of the effect of sonification on the perception of movements, fMRI activations to short video segments showing an avatar performing breaststroke movements accompanied either by congruent sounds, generated from kinematic parameters of the visual stimuli, or by incongruent sounds were studied in normal healthy volunteers. As in the behavioral experiment, participants had to compare two successive short video segments of a trial with regard to movement speed.

In addition to standard univariate analyses, fMRI was also analyzed using connectivity analysis [17]. We hypothesized that congruently sonified movements would engage additional brain areas relative to incongruent stimuli and that this network should, at least in part, coincide with brain areas identified as important for audiomotor integration.

\section{Methods}

All procedures had been cleared by the ethics committee of the University of Magdeburg, the affiliation of the corresponding author at the time of the study.

\section{Participants}

Seventeen student volunteers from different fields of study (7 women, age 24.6 years \pm 4.4 ). At the time of testing none of the participants practiced swimming on a regular basis. Formerly, participants had engaged in regular swimming for 3.2 years (SD 4.1). Also, none of the participants could be considered expert musicians. Six of the participants never had learned to play an instrument. The mean number of years of active playing was 5.5 years (SD 6.1). All participants were healthy, right-handed native speakers of German with no history of neurological or psychiatric impairments. Basic visual 
and auditory abilities were normal as tested using a standard vision test for acuity and audiometry.

The subjects participated in a first behavioral session (I) and a second refreshing behavioral session (II) about five weeks later immediately prior to the fMRI session.

\section{Stimulus material}

Behavioral as well as fMRI stimulus material was nearly identical, only differing in duration and inter-stimulus -interval.

The visual stimulus component comprised a solid swimmer model performing breaststroke movements (Figure 1). Kinematics of the model were based on real human motion data and had been derived from 3D-video captures of a former breaststroke world champion. Absolute motion was eliminated by keeping the centre of the pelvis stationary. Therefore only relative motion was displayed. The congruent auditory stimulus component consisted of a movement sonification based on two kinematic parameters of the visual model: First, relative distance of the wrist joints to the centre of the pelvis was mapped to frequency of an electronic sound called "Fairlight Aahs". Moreover the relative velocity of this movement component was mapped to the loudness of the "Fairlight Aahs". Both, velocity and loudness represent a joint intermodal elementary intensity category. The range of frequency modulation ("pitching") covered the interval between fis' and e". Second, the relative distance of the ankle joints to the centre of the pelvis was mapped to the frequency of an electronic sound called "Pop Oohs". Again, the velocity of the movement was represented by the loudness of the sound. The pitch range covered the interval between contra $\mathrm{B}^{\prime}$ and $\mathrm{D}$. Both sounds were selected from the 'E-MU E4K' sound library. The kinematic-acoustic mapping was realized by using the 'Sonification-Tool'-Software [18] and provided a high degree of visual auditory stimulus convergence.

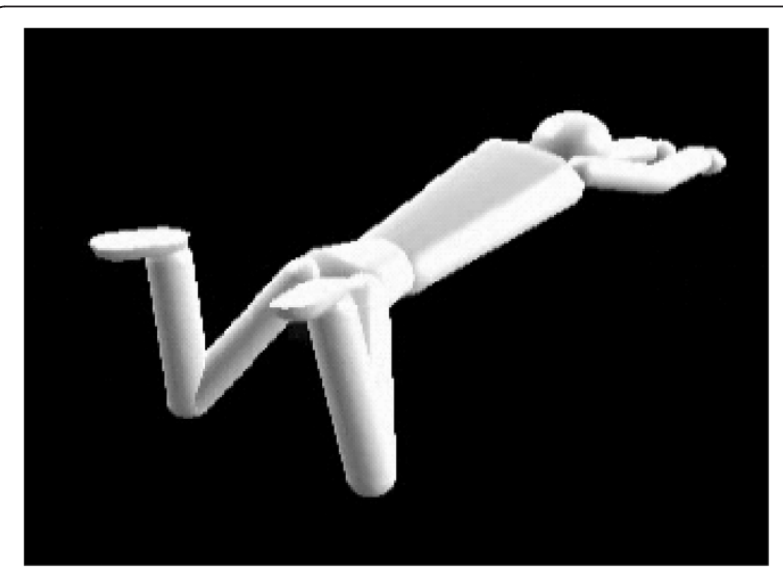

Figure 1 Stimuli. Visual stimulus component: a model of a solid swimmer performing breaststroke movements.
Incongruent auditory information featured two different chords covering a similar timbre and pitch range as the congruent sonification over the course of a breaststroke. One chord lasted $1.0 \mathrm{~s}, 1.32 \mathrm{~s}, 1.8 \mathrm{~s}$ or $2.0 \mathrm{~s}$ and then changed into a second chord. As any kind of correspondence between chord switching and movement kinematics was avoided, the incongruent auditory information does not meet any criteria of a sonification. Details about the auditory part of the stimuli are given in Figure 2.

Original relative velocity of the audiovisual stimuli (100\%) was varied in five steps (98\%, 94\%, 92\%, 90\% and $88 \%$ ) to achieve subtle temporal variations of the swimming frequency. Those temporal variations were reduced to $98 \%, 94 \%$ and $92 \%$ in the fMRI session due to task requirements. The original kinematic data were interpolated and visualized with the 'Simba 2.0' Software to keep temporal continuity. Identical temporal variation was applied to the auditory stimuli: Sound sequences were stretched to $98 \%, 94 \%, 92 \%, 90 \%$ and $88 \%$ of the origin with 'cool edit 2.0' Software. Pitch frequency was preserved on stretching in order to enhance discrimination difficulty. To keep consistency of kinematicacoustical mapping on the other hand - relative velocity of the swimmer model was mapped to sound amplitude and pitch frequency - pitch frequency was subsequently transposed marginally to $99 \%, 97 \%, 96 \%, 95 \%$ and $94 \%$ of the original.

\section{Procedure}

A single trial consisted of two consecutive stimuli. Each stimulus contained of about five cycles of breast stroking in the behavioral session and was reduced to about two and a half cycles in the fMRI scanner session due to the temporal limitations of imaging studies. The duration of a single breast stroke cycle (at 100\%) was $1.12 \mathrm{~s}$. Absolute duration of a single stimulus was standardized to $6 \mathrm{~s}$ for the behavioral session and $3 \mathrm{~s}$ for the imaging session. The posture of the swim model at the first and the last picture of each stimulus was randomly varied to prevent an identification of a distinct stimulus based on initial and/or final posture. The inter-stimulus interval was set to $1.5 \mathrm{~s}$ (behavioral) or $0.5 \mathrm{~s}$ (imaging). The inter-trial interval lasted $6 \mathrm{~s}$, providing $5 \mathrm{~s}$ for verbal response and $1 \mathrm{~s}$ for the indication to the next trial by presenting the trial number in the behavioural study. Inter-trial-interval was $11.5 \mathrm{~s}$ in the fMRI session allowing for the decline of the BOLD signal. In the fMRI study a manual response (pressing one of two buttons on an MRI congruent response pad) rather than a verbal response was used.

In behavioral session I the visual stimuli were projected on a $2.30 * 1.70 \mathrm{~m}$ sized screen located $4 \mathrm{~m}$ in front of the participants. In session II visual stimuli were 


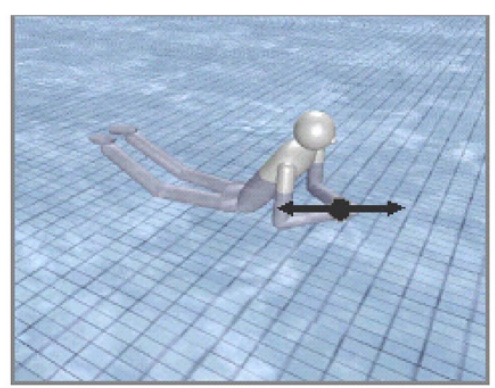

arm cycle
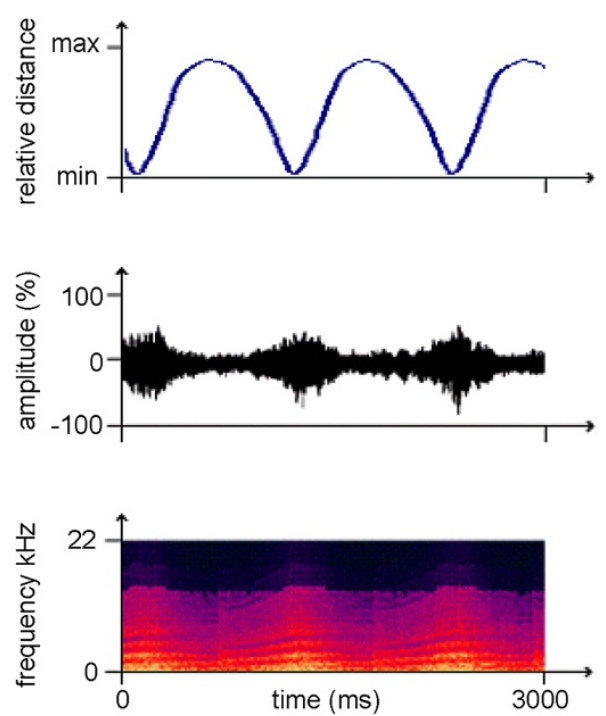

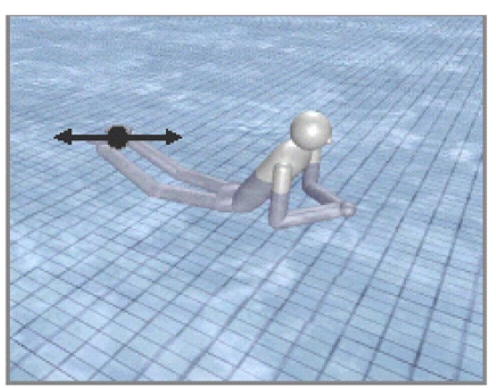

leg cycle
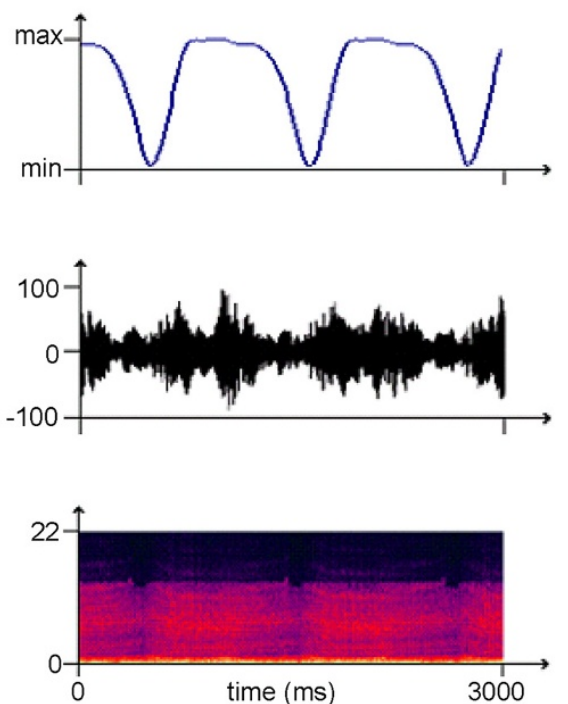

Figure 2 Kinematic-acoustic mapping. In the congruent condition frequency and amplitude modulations of electronic sounds represented changes in the relative distance between the wrist joints ("arm cycle", top and second row left) or the ankle joints ("leg-cycle", top and second row right) to the center of the pelvis. Third row: Sound pressure diagram; Fourth row: Spectrogram. Amplitude is color coded with cold / hot colors denoting low / high amplitudes.

displayed on a $0.37 * 0.23 \mathrm{~m}$ sized video-screen $0.5 \mathrm{~m}$ in front of the participants. Auditory stimuli were presented via headphones (beyerdynamic DT 100). Congruent and incongruent stimuli were arranged in blocks of 26 (session I) or 13 (session II) trials each. To investigate the perceptual effects of movement sonification, participants were instructed to estimate differences of swimming velocities between two consecutive breaststroke sequences. The mean absolute error (AE) of the absolute difference between the participants' verbal response and the actual temporal difference of four breaststroke cycles from two consecutive sequences was chosen as dependent variable.

In the fMRI session visual stimuli were presented via MR-congruent video-goggles and the sound stimuli were presented by a shielded pneumatic headphone system with the sound level adapted such to be clearly audible against the scanner noise. The fMRI task required participants to judge whether the swimming velocities of stimulus 1 and 2 of a trial were "same" or "different" by pressing one of two buttons with the thumb of their right hand. A factorial design crossing the factors audiovisual congruency (congruent vs. incongruent) and velocity (same vs. different) was used. Twenty-four trials were presented for each of the 4 resulting conditions in random order.

\section{FMRI data acquisition and analysis}

Data were collected on a 3-T Siemens Allegra system. Functional images were acquired using a $\mathrm{T} 2{ }^{*}$ weighted echo planar imaging (EPI) sequence, with 2000-ms time repetition (TR), 30-ms time echo (TE), and $80^{\circ}$ flip angle, in four runs. Each functional image consisted of 30 axial slices, with $64 * 64$ matrix, $220 \mathrm{~mm} * 220 \mathrm{~mm}$ field of view (FOV), $3.5-\mathrm{mm}$ thickness, $0.35-\mathrm{mm}$ gap, and $3.5 \mathrm{~mm} * 3.5 \mathrm{~mm}$ in-plane resolution.

Structural images were acquired using a T1-weighted magnetization-prepared rapid-acquired gradient echo (MPRAGE) sequence, with 2500-ms TR, 1.68-ms TE, and $7^{\circ}$ flip angle. The structural image consisted of 192 
slices, with 256*256 matrix, $256 \mathrm{~mm}^{*} 256 \mathrm{~mm}$ FOV, 1 -mm thickness, no gap, and $1 \mathrm{~mm}^{* 1} \mathrm{~mm}$ in-plane resolution.

Data were analyzed with SPM8 (http://www.fil.ion.ucl. ac.uk/spm). The first four volumes were discarded owing to longitudinal magnetization equilibration effects. Functional images were first time-shifted with reference to the middle slice to correct differences in slice acquisition time. They were then realigned with a least squares approach and a rigid body spatial transformation to remove movement artifacts. Estimated movement parameters (six parameters per image: $x, y, z$, pitch, roll, and yaw) were included in GLMs as nuisance regressors of no interest to minimize signal-corrected motion effects. Realigned images were normalized to the EPI-derived MNI template (ICBM 152, Montreal Neurological Institute) and resampled to $2 \mathrm{~mm} \times 2 \mathrm{~mm} \times 2 \mathrm{~mm}$ voxel. Normalized images were smoothed with a Gaussian kernel of $8-\mathrm{mm}$ full-width half-maximum (FWHM) and filtered with a high-pass filter of $128 \mathrm{~s}$.

We carried out two statistical analyses, i.e. a standard univariate analysis and a functional connectivity analysis.

\section{Standard univariate analysis}

The standard univariate analysis was performed to examine brain regions differentially activated in the processing of 'congruent' vs. 'incongruent' stimuli. Moreover, we also examined the effect of matching and nonmatching stimulus pairs. This analysis was implemented on the basis of a GLM by using one covariate to model hemodynamic responses of all stimuli of a condition. Classical parameter estimation was applied with a onelag autoregressive model to whiten temporal noise in fMRI time courses of each participant in order to reduce the number of false-positive voxels. The contrast maps were entered into two one-sample $t$ tests on the group level. Resulting activation maps were considered at $p<0.05$ (FDR-corrected) with a minimum cluster size of 10 voxels.

\section{Functional connectivity analysis}

The functional connectivity analysis was performed to examine interregional interactions modulated in the processing of 'congruent' and 'incongruent' stimuli. This analysis was implemented on the basis of a GLM by using separate covariates to model hemodynamic responses of each single stimulus in each condition. Classical parameter estimation was applied with a one-lag autoregressive model. For each participant, estimated beta values were extracted to form a set of condition-specific beta series. The left STS (defined as a sphere of $5 \mathrm{~mm}$ around the activation peak in the univariate analysis) was defined as a seed region. Beta series of each seed were averaged across voxels within the critical region and correlated with beta series of every other voxel in the whole brain. Maps of correlation coefficients were calculated for each participant in each condition. The correlation maps were normalized with an arc-hyperbolic tangent transform and entered into two paired-sample $t$ tests on the group level. Resulting connection maps were considered at $p<0.05$ (FDR-corrected) with a minimum cluster size of 100 voxels. Two further seed regions were defined (right Brodmann area 6, right Brodmann area 44) but results will not be reported in this paper.

\section{Results}

\section{Behavioral results}

The results of the two behavioral sessions are shown in Figure 3. AE was significantly lower in the congruent than the incongruent condition as confirmed by a two-way ANOVA with a significant effect condition $\left(\mathrm{F}_{(1,16)}=25.93\right.$, $\left.\mathrm{p}<0.001, \quad \eta_{\mathrm{p}}{ }^{2}=0.62\right)$. Neither differences between both sessions nor the interaction were significant (session: $\mathrm{F}_{(1,16)}=1.70, \mathrm{p}>0.05, \eta_{\mathrm{p}}{ }^{2}=0.10$; session*condition: $\mathrm{F}_{(1,16)}=1.59$, $\left.\mathrm{p}>0.05, \eta_{\mathrm{p}}{ }^{2}=0.09\right)$. Therefore congruent audiovisual information led to more accurate perceptual judgements than incongruent audiovisual information.

\section{Imaging results}

The results of the univariate analysis are shown in Figure 4A and Table 1. Congruent stimuli led to enhanced activity in superior and medial posterior temporal regions as well as the insula bilaterally and the precentral gyrus on the right side. Incongruent stimuli on the other hand were associated with more activity in the inferior temporal cortex (left), the frontal operculum (right), Brodmann area 6 (left) and the inferior parietal lobule. We also assessed activation differences between the congruent stimuli in which the two segments had

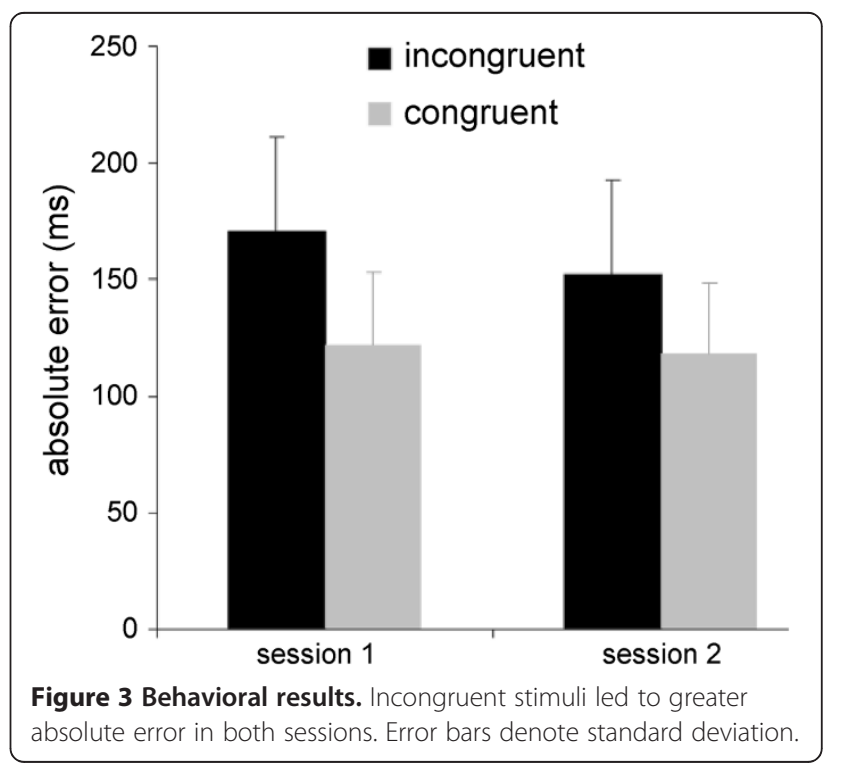




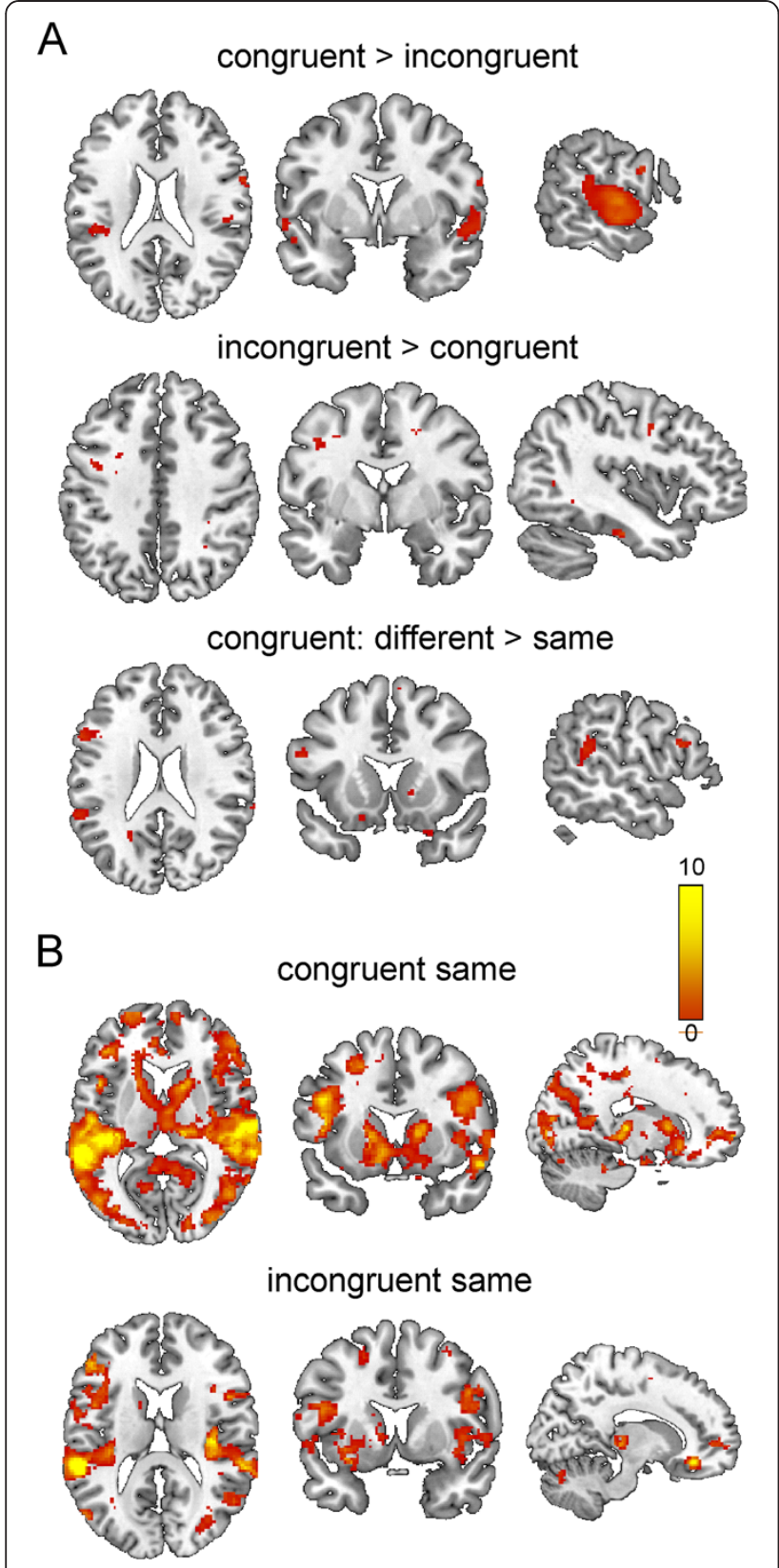

Figure 4 fMRI results. A: univariate analysis. FDR-corrected, $p<0.05$, minimum cluster size 10 voxels. B: Connectivity analysis using the left STS as a seed. For congruent stimuli more widespread connectivity is observed including frontal and parietal cortical areas as well as thalamus, caudate nucleus and putamen. FDR-corrected, $p<0.05$, minimum cluster size 100 voxels.

different speeds vs. same speed. The former stimuli led to more activation in a number of brain areas as summarized in Table 1 and Figure 4A (bottom panel).

To assess the influence of sonification on network activity, connectivity analysis was performed using the left STS as a seed region separately for congruent and incongruent stimuli (same speed trials, Figure 4B, Tables 2
Table 1 Univariate analysis

\begin{tabular}{|c|c|c|c|c|c|c|c|}
\hline Region & $\mathrm{BA}$ & $\mathrm{H}$ & $\mathrm{X}$ & $Y$ & Z & $t$ & Size \\
\hline \multicolumn{8}{|c|}{ congruent > incongruent } \\
\hline Sup \& mid temporal, insula & 21 & $L$ & -62 & -8 & -26 & 6.26 & 1955 \\
\hline Sup \& mid temporal, insula & 22 & $\mathrm{R}$ & 60 & -10 & 2 & 6.81 & 2095 \\
\hline Precentral & 6 & $\mathrm{R}$ & 62 & 4 & 24 & 5.06 & 23 \\
\hline \multicolumn{8}{|c|}{ Incongruent > congruent } \\
\hline Inferior temporal & 20 & $L$ & -42 & -18 & -26 & 5.25 & 17 \\
\hline \multirow[t]{2}{*}{ Frontal operculum } & 44 & $\mathrm{R}$ & 44 & 6 & 22 & 4.20 & 29 \\
\hline & 6 & $L$ & -42 & 0 & 40 & 4.43 & 20 \\
\hline Inf parietal lobule & 40 & $\mathrm{R}$ & 48 & 42 & 46 & 4.49 & 19 \\
\hline \multicolumn{8}{|c|}{ congruent: different > same } \\
\hline Superior temporal & 21 & $\mathrm{R}$ & 66 & -12 & -8 & 4.99 & 24 \\
\hline Medial temporal & 21 & $\mathrm{R}$ & 52 & -32 & -2 & 4.35 & 27 \\
\hline Inferior frontal gyrus & 45 & $\mathrm{R}$ & -44 & 30 & 2 & 4.34 & 30 \\
\hline Sup \& mid temporal & 48 & $L$ & -62 & -42 & 28 & 4.86 & 198 \\
\hline Insula & 48 & $\mathrm{R}$ & 34 & -18 & 10 & 4.88 & 35 \\
\hline Inferior frontal gyrus & 44 & $L$ & -56 & 18 & 20 & 5.05 & 54 \\
\hline Cingulate gyrus & - & $L$ & -14 & -22 & 36 & 6.50 & 134 \\
\hline Precentral & 6 & $\mathrm{R}$ & 46 & -12 & 56 & 4.70 & 54 \\
\hline Postcentral & 4 & $L$ & -18 & -36 & 72 & 4.66 & 24 \\
\hline
\end{tabular}

$B A$, Brodmann Area; $H$, hemisphere; coordinates in MNI; $t$, statistic values; $L$, left; $R$, right; Size, number of voxels.

and 3). Clearly different connectivity patterns emerged for the congruent and incongruent stimuli. Whereas for congruent stimuli pronounced connectivity of the STS with the basal ganglia and thalamus as well as frontal regions was observed, this was not seen to the same extent for the incongruent stimuli.

We also performed connectivity analyses using the right BA6 and the right BA44 as seed regions. The results are illustrated in Figure 5. The connectivity patterns obtained for these seed regions also revealed differences for congruent and incongruent stimuli. For the former, increased connectivity to basal ganglia and motor cortical areas was observed for congruent stimuli. This was more prominent for the Brodmann area 44 seed.

\section{Discussion}

The present study asked two main questions: (a) To what extent congruent sonification accompanying movements improves perceptual processing of these movements, and (b) What are the brain systems supporting the processing of sonified movements?

The first question was addressed by the behavioural part of the study. Clearly, sonification led to a decisive advantage in the perceptual judgement task in that the errors associated with the comparison of the movement speed of the two video-segments of a trial were 
Table 2 Connectivity analysis, seed left STS, condition congruent / same

\begin{tabular}{|c|c|c|c|}
\hline Cluster 1 & xyz & Region & Size (voxel) \\
\hline$T=22.18$ & $-56-3812$ & left superior temporal cortex & 37455 \\
\hline subcluster & sum classified voxels & \multicolumn{2}{|l|}{ subregion } \\
\hline frontal & 4909 & \multicolumn{2}{|c|}{ 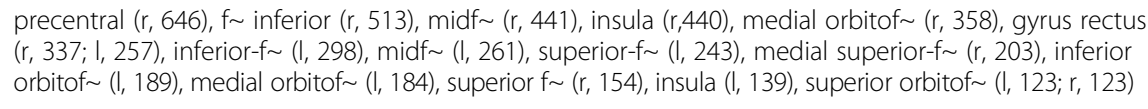 } \\
\hline temporal & 2672 & \multicolumn{2}{|c|}{$\begin{array}{l}\text { rolandic operculum }(r, 455) \text {, inferior } t \sim(l, 375), t \sim \text { pole }(r, 307) \text {, inferior } t \sim(r, 300) \text {, rolandic operculum } \\
(l, 202) \text {, hippocampus }(r, 184) \text {, parahippocampus }(r, 167 ; l, 139) \text {, angularis }(r, 122) \text {, Heschl }(r, 237 ; l, 184)\end{array}$} \\
\hline occipital & 1989 & \multicolumn{2}{|c|}{ superior o (l, 631), calcarine sulcus $(r, 319 ; 1,260)$, lingual $(l, 249)$, inferior o (l, 204; r 192), lingual $(r, 134)$} \\
\hline fusiform/cuneus & 1578 & \multicolumn{2}{|c|}{ fusiform $(I, 502)$, cuneus $(l, 429 ; r, 359)$, fusiform $(r, 288)$, } \\
\hline cingulate gyrus & 1494 & \multicolumn{2}{|c|}{ mid $c \sim(r, 584)$, anterior $c \sim(1,516)$, posterior $c \sim(1,228 ; r, 166)$} \\
\hline parietal & 1445 & \multicolumn{2}{|c|}{ superior $p \sim(1,364 ; r, 324)$, inferior $p \sim(1,303)$, supra marginalis $(I, 204 ; r, 125)$, inferior $p \sim(r, 125)$} \\
\hline thalamus & 695 & \multicolumn{2}{|l|}{ thalamus $(r, 482 ; 1,213)$} \\
\hline caudatus/putamen & 684 & \multicolumn{2}{|c|}{ caudatus $(I, 438)$, putamen $(I, 246)$, } \\
\hline cerebellum & 186 & \multicolumn{2}{|l|}{ cerebellum $(\mathrm{l}, 186)$} \\
\hline Cluster 2 & $x y z$ & region & size (voxel) \\
\hline$T=10.18$ & -461026 & left frontal inferior cortex & 2528 \\
\hline subcluster & sum classified voxels & \multicolumn{2}{|l|}{ subregion } \\
\hline frontal & 1714 & \multicolumn{2}{|c|}{ mid $f \sim(l, 747)$, precentral $(l, 400)$, inferior $f \sim(1,355)$, superior $f \sim(l, 212)$} \\
\hline parietal & 218 & \multicolumn{2}{|l|}{ postcentral $(\mathrm{l}, 218)$} \\
\hline Cluster 3 & $x y z$ & region & size (voxel) \\
\hline $\mathrm{T}=7.48$ & 26252 & Left mid frontal cortex & 236 \\
\hline Cluster 4 & $x y z$ & region & size (voxel) \\
\hline $\mathrm{T}=6.72$ & $8-28-28$ & Right cerebellum & 107 \\
\hline subcluster & sum classified voxels & subregion & \\
\hline Cerebellum/ brainstem & 107 & pons (90), cerebellum $(\mathrm{l}, 17)$ & \\
\hline
\end{tabular}

$T$, statistic values; l, left; $r$, right; size, number of voxels, $c \sim$ cingulate, $f \sim$ frontal, $t \sim$ temporal, $p \sim$ parietal, xyz coordinates of the peak voxel (MNI space).

considerably smaller for congruent stimuli. Shams and Seitz [14] argued that, whereas "training on any pair of multisensory stimuli might induce a more effective representation of the unisensory stimulus, the effects could be substantially more pronounced for congruent stimuli." They defined congruency as supported by "relationships between the senses found in nature. This spans the basic attributes such as concordance in space and time, in addition to higher-level features such as semantic content (e.g. object and speech information)." Indeed, in a perceptual learning experiment, in which one group was trained with congruent auditory-visual moving stimuli, the second group with incongruent auditoryvisual stimuli and the third group with visual stimuli only, facilitation was specific to the congruent condition, thus ruling out a general alerting effect of the additional auditory stimulus [19]. The highly significant effect of congruency in the present study is a further proof for the benefit brought about by additional congruent sonification. It has to be kept in mind, however, that the present study used realistic biological motion stimuli with sonification based on kinematic parameters, whereas Kim et al. required the detection of coherently moving dots that were displaced and accompanied by a similar displacement of sound direction.

\section{fMRI}

With regard to the neural underpinnings of the facilitatory effect of congruency fMRI showed marked differences between congruent and incongruent stimuli. The univariate analysis showed increased activation for congruent relative to incongruent stimuli in the superior and medial posterior temporal regions as well as the insula bilaterally and the precentral gyrus on the right side. The superior temporal region has been shown to be involved in multisensory processing in multiple studies. It receives converging auditory and visual inputs [20] and thus is equipped to contribute to multisensory integration [21-24]. Noesselt et al. [25] investigated trains of auditory and visual stimuli that either coincided in time or not. These authors found increased activation in STS when the visual stream coincided in time with the auditory stream and decreased activation for noncoincidence (using activation to unisensory stimuli as 
Table 3 Connectivity analysis, seed left STS, condition incongruent / same

\begin{tabular}{|c|c|c|c|}
\hline Cluster 1 & $x y z$ & Region & size (voxel) \\
\hline$T=21.88$ & $-56-3812$ & left superior temporal cortex & 4837 \\
\hline subcluster & sum classified voxels & \multicolumn{2}{|l|}{ subregion } \\
\hline frontal & & \multicolumn{2}{|c|}{ inferior $f \sim(l, 377)$, insula $(l, 319)$, frontal inferior operculum $(l, 269)$} \\
\hline temporal & & \multicolumn{2}{|c|}{ superior $t \sim(\mid, 1453)$, mid $t \sim(\mid, 663)$, rolandic operculum $(I, 311)$} \\
\hline parietal & & \multicolumn{2}{|c|}{ supra marginalis $(I, 214)$, Heschl $(I, 122)$} \\
\hline caudatus/putamen & & \multicolumn{2}{|l|}{ putamen $(\mid, 115)$} \\
\hline Cluster 2 & $x y z$ & region & size (voxel) \\
\hline $\mathrm{T}=14.86$ & $58-16-4$ & left mid temporal cortex & 4566 \\
\hline subcluster & sum classified voxels & \multicolumn{2}{|l|}{ subregion } \\
\hline temporal & 2096 & \multicolumn{2}{|c|}{ superior $t \sim(r, 1491)$, Heschl $(r, 214)$, mid- $t \sim(r, 200)$, rolandic operculum $(r, 191)$} \\
\hline frontalparietal & 758 & \multicolumn{2}{|l|}{ insula $(r, 758)$} \\
\hline Cluster 3 & $x y z$ & region & size (voxel) \\
\hline$T=12.23$ & $1034-14$ & left gyrus rectus & 713 \\
\hline subcluster & sum activated voxels & \multicolumn{2}{|l|}{ subregion } \\
\hline frontal & 272 & \multicolumn{2}{|l|}{ medial orbitof $(\mathrm{l}, 147 ; \mathrm{r}, 125)$} \\
\hline cingulate gyrus & 110 & \multicolumn{2}{|l|}{ anterior $c \sim(r, 110)$} \\
\hline Cluster 4 & $x y z$ & \multicolumn{2}{|l|}{ region } \\
\hline$T=10.15$ & $-54-7020$ & left mid temporal cortex & 377 \\
\hline subcluster & sum classified voxels & \multicolumn{2}{|l|}{ subregion } \\
\hline occipital & 228 & \multicolumn{2}{|l|}{ mid o (l, 228) } \\
\hline temporal & 91 & \multicolumn{2}{|l|}{ mid $t \sim(\mid, 91)$} \\
\hline \multicolumn{4}{|l|}{ Cluster 5} \\
\hline $\mathrm{T}=7.43$ & $46-5814$ & right mid temporal cortex & 317 \\
\hline \multicolumn{4}{|l|}{ Cluster 6} \\
\hline $\mathrm{T}=8.52$ & $42-56-10$ & right inferior temporal cortex & 226 \\
\hline \multicolumn{4}{|l|}{ Cluster 7} \\
\hline$T=6.05$ & $36-7218$ & right mid temporal cortex & 197 \\
\hline \multicolumn{4}{|l|}{ Cluster 8} \\
\hline $\mathrm{T}=6.78$ & $6-58-30$ & cerebellum & 131 \\
\hline \multicolumn{4}{|l|}{ Cluster 9} \\
\hline $\mathrm{T}=8.37$ & $-34-14-22$ & right hippocampus & 106 \\
\hline subcluster & sum classified voxels & \multicolumn{2}{|l|}{ subregion } \\
\hline temporal & 48 & \multicolumn{2}{|l|}{ hippocampus $(1,48)$} \\
\hline fusiform/cuneus & 21 & \multicolumn{2}{|l|}{ fusiform $(\mathrm{l}, 21)$} \\
\hline
\end{tabular}

$T$, statistic values; l, left; $r$, right; size, number of voxels, $c \sim$ cingulate, $f \sim$ frontal, $t \sim$ temporal, $p \sim$ parietal, xyz coordinates of the peak voxel (MNI space).

baseline). An influence of audiovisual synchrony has also been found in a number of other fMRI studies [26-29]. With regard to the audiovisual integration of speech stimuli for which the synchrony of lip-movements and sounds is of great importance again the caudal part of the superior temporal sulcus has been implicated $[24,30,31]$. A number of studies have revealed activation for audiovisual speech stimuli compared to their unimodal components presented separately [32,33]. It has further been shown that the visual component of audiovisual speech stimuli exerts a modulatory influence on the auditory areas located in the dorsal surface of the temporal lobe $[34,35]$.

In light of these previous findings the increased activation in the superior temporal region for congruent stimuli in the univariate analysis suggests that audiovisual congruency leads to engagement of multisensory integration areas. This notion is further substantiated by the connectivity analysis (Figure 4B). Placing a seed in the left STS region revealed a widespread connectivity 


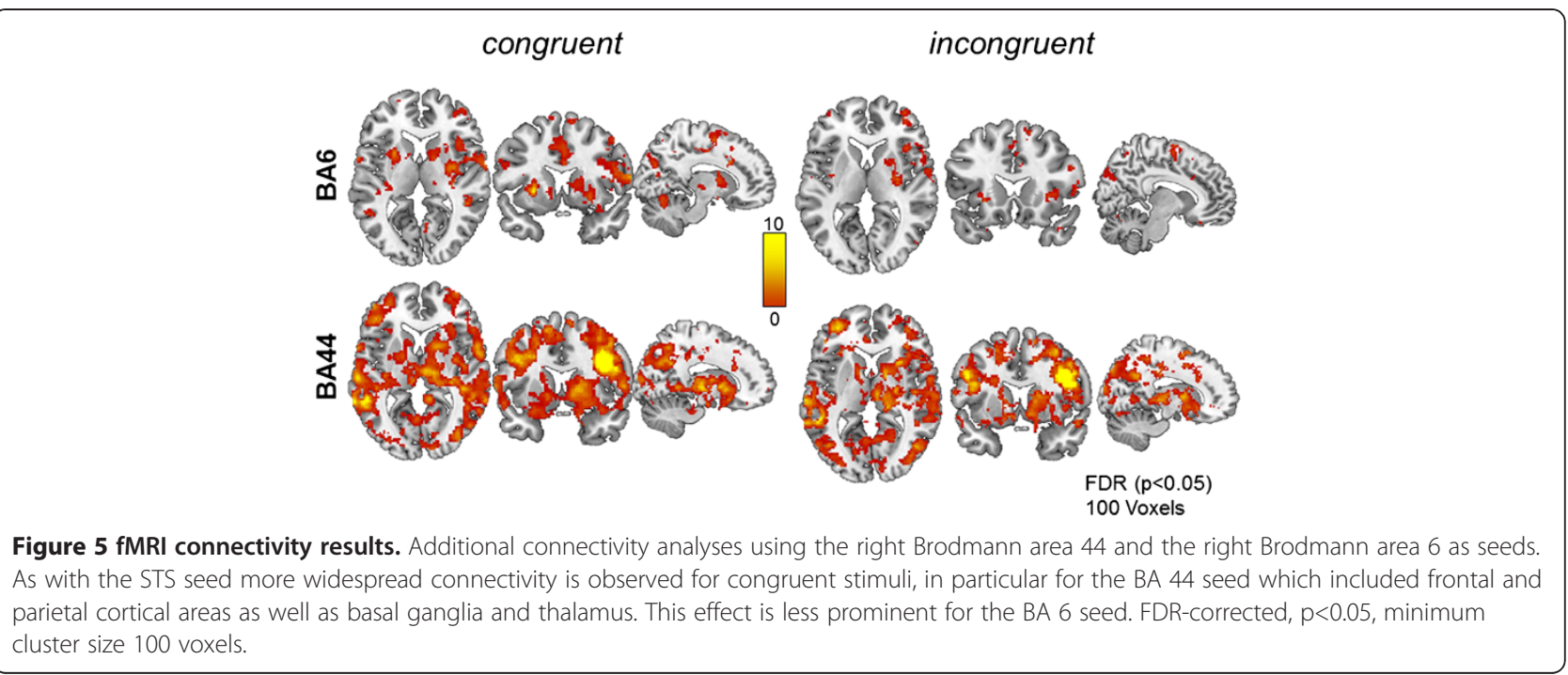

pattern for the congruent stimuli: Besides subcortical key players of the striato-thalamo-frontal motor-loops such as the caudate nucleus, putamen, thalamus and cerebellum, this network also included cortical regions in the medial superior frontal gyrus, superior, middle and inferior frontal gyrus, cingulate cortex, pre- and postcentral gyrus and parietal areas. By contrast, the incongruent stimuli engaged a much less widespread network. In particular, no connectivity was observed between the STS and the caudate nucleus and the putamen and the connectivity to the thalamus and cerebellum was less pronounced in comparison to the congruent stimuli. Also, with regard to cortical regions, incongruent stimuli showed a greatly reduced connectivity to frontal areas. This increased recruitment of basal ganglia and frontal motor-related areas was also seen for two additional seed areas (right Brodmann areas 6 and 44, Figure 5).

We would like to discuss the current patterns with regard to two topics: action observation and audiovisual integration. It has been proposed that the brain of an observer who observes someone else performing an action may simulate the performance [36] using a special neural system that has been termed the mirror neuron system [37-43]. The classical studies by Rizzolatti's group have shown that the premotor and parietal cortex of monkeys harbours mirror neurons which discharge not only when the monkey performs an action but also when the monkey observes another monkey or an experimenter performing the same action $[40,41,44]$. Numerous brain imaging studies have suggested that a similar mirror neuron system exists in humans and comprises premotor cortex, parietal areas and the superior temporal sulcus (STS) [38,45-50]

With regard to the stimuli of the current study it is important that while observing the actions of an artificial handled to less activation of the mirror system than watching real hand actions [51,52], biomechanically possible actions (as used in the present study) give rise to robust activations compared to impossible movements [53]. Systematic manipulation of the stimuli further suggests that the human mirror system reflects the overlap between an observed action and the motor repertoire of the observer [54].

The current study revealed robust activation of major hubs of the human action observation system. In particular, the connectivity analysis showed that the STS during observation of the breast-stroking movement was intimately connected to frontal (including Brodmann areas 44 and 45) and parietal cortical areas that have been previously found in relation to action observation.

Importantly, we also found that congruent sonification compared to incongruent concurrent sounds led to increased activation in parts of the mirror neuron system including the frontal operculum, inferior parietal lobule and the superior temporal areas. The superior temporal area has been identified as being important for a number of complex cognitive processes: It has been found active during the processing of biological motion $[55,56]$ and, emanating from this more basic capability, social perception [57-59]. As pointed out in the introduction, it has also been identified as important for audiovisual integration [25,60-62]. An integrative view of the functions of this area has been provided by Hein and Knight [63]. What is more, the connectivity analysis using the left STS as a seed region revealed a more robust and widespread connectivity for congruent compared to incongruent stimuli. Interestingly, trials with congruent sonification also showed connectivity to subcortical structures known to be part of the striato-thalamofrontal motor loops, i.e. the caudate nucleus, putamen and the thalamus. 


\section{Conclusion}

This suggests that congruent sonification amplifies the neural activity of the action observation system. As shown in the behavioural part of this study, this enhanced neural representation of the observed movement leads to an improved perceptual analysis of the movement. Experiences in sports science also indicate that sonification of movements during exercise also results in improved, more precise performance of complex movements, such as rowing, golf driving, hammer throwing or swimming [12,64-69]. Further research needs to address whether athletes trained using movement sonification possess an enhanced representation of movements similar to professional musicians $[4-7,70]$.

\section{Abbreviations}

AE: Absolute error; BOLD: Blood oxygen level dependent; EPI: Echo planar imaging; fMRI: Functional magnetic resonance imaging; GLM: General linear model; STS: Superior temporal sulcus.

\section{Competing interests}

The authors declare that they have no competing interests.

\section{Authors' contributions}

GS, AE, TFM conceived the experimental design. BM, GS, AS performed the experiments. BM, AS, GS, AE, MH, TFM performed the analyses. AH and GS constructed the stimuli and the stimulation scenarios. GS, AE, TFM and BM wrote the various drafts of the manuscript. All authors read and approved the final manuscript.

\section{Acknowledgements}

This research was supported by the Deutsche Forschungsgemeinsschaft (DFG, SFB TR31, TP A7).

\section{Author details \\ ${ }^{1}$ Institute of Sports Science, University of Hannover, Hannover, Germany. ${ }^{2}$ Department of Neurology, University of Lübeck, Lübeck, Germany. ${ }^{3}$ International Neuroscience Institute, Hannover, Germany. ${ }^{4}$ Department of Psychiatry, University of Erlangen, Erlangen, Germany.}

Received: 28 August 2012 Accepted: 7 March 2013

Published: 14 March 2013

\section{References}

1. Haueisen J, Knösche TR: Involuntary motor activity in pianists evoked by music perception. J Cogn Neurosci 2001, 13:786-792.

2. Lotze M, Scheler G, Tan HRM, Braun C, Birbaumer N: The musician's brain: Functional imaging of amateurs and professionals during performance and imagery. Neuroimage 2003, 20:1817-1829.

3. Meister IG, Krings T, Foltys H, Boroojerdi B, Müller M, Töpper R, Thron A: Playing piano in the mind - An fMRI study on music imagery and performance in pianists. Cogn Brain Res 2004, 19:219-228.

4. Baumann O, Greenlee MW: Neural correlates of coherent audiovisual motion perception. Cereb Cortex 2007, 17:1433-1443.

5. Baumann S, Koeneke S, Schmidt CF, Meyer M, Lutz K, Jancke L: A network for audio-motor coordination in skilled pianists and non-musicians. Brain Res 2007, 1161:65-78.

6. Haslinger B, Erhard P, Altenmüller E, Schroeder U, Boecker H, Ceballos-Baumann AO: Transmodal sensorimotor networks during action observation in professional pianists. J Cogn Neurosci 2005, 17:282-293.

7. Bangert $M$, Peschel $T$, Schlaug G, Rotte $M$, Drescher D, Hinrichs $H$, Heinze $H$ J, Altenmuller E: Shared networks for auditory and motor processing in professional pianists: evidence from fMRI conjunction. Neuroimage 2006 30:917-926.

8. Scheef $\mathrm{L}$, Boecker H, Daamen M, Fehse U, Landsberg MW, Granath DO, Mechling $\mathrm{H}$, Effenberg AO: Multimodal motion processing in area V5/MT: evidence from an artificial class of audio-visual events. Brain Res 2009, 1252:94-104

9. Altenmüller E, Marco-Pallares J, Münte TF, Schneider S: Neural reorganization underlies improvement in stroke-induced motor dysfunction by music-supported therapy. Ann N Y Acad Sci 2009, 1169:395-405.

10. Schneider S, Münte T, Rodriguez-Fornells A, Sailer M, Altenmüller E: Musicsupported training is more efficient than functional motor training for recovery of fine motor skills in stroke patients. Music Perception 2010, 27:271-280.

11. Schneider S, Schönle PW, Altenmüller E, Münte TF: Using musical instruments to improve motor skill recovery following a stroke.

J Neurol 2007, 254:1339-1346.

12. Effenberg AO: Movement sonification: Effects on perception and action. IEEE Multimedia 2005, 12:53-59.

13. Effenberg $A O$, Mechling $\mathrm{H}$ : Movement-sonification: $\mathrm{A}$ new approach in motor control and learning. J Sports Exercise Psychol 2005, 27:58-68.

14. Shams L, Seitz AR: Benefits of multisensory learning. Trends Cogn Sci 2008, 12:411-417.

15. Seitz AR, Kim R, Shams L: Sound Facilitates Visual Learning. Curr Biol 2006, 16:1422-1427.

16. Chollet D, Madani M, Micallef JP: Effects of two types of biomechanical bio-feedback on crawl performance. In Biomechanics and Medicine in Swimming, Swimming Science VI. Edited by MacLaren D, Reilly T, Lees A. Cambridge: SPON Press; 1992:48-53.

17. Rissman J, Gazzaley A, D'Esposito M: Measuring functional connectivity during distinct stages of a cognitive task. Neuroimage 2004, 23:752-763.

18. Becker A: Echtzeitverarbeitung dynamischer Bewegungsdaten mit Anwendungen in der Sonification. Bonn: University of Bonn; 1999.

19. Kim RS, Seitz AR, Shams L: Benefits of stimulus congruency for multisensory facilitation of visual learning. PLOS One 2008, 3:e1532.

20. Kaas $\mathrm{JH}$, Collins CE: The resurrection of multisensory cortex in primates. In The Handbook of Multisensory Processes. Edited by Calvert GA, Spence S, Stein BE. Cambridge: MIT Press; 2004:285-293.

21. Benevento LA, Fallon J, Davis BJ, Rezak M: Auditory visual interaction in single cells in the cortex of the superior temporal sulcus and the orbital frontal cortex of the macaque monkey. Exp Neurol 1977, 57:849-872.

22. Bruce C, Desimone R, Gross CG: Visual properties of neurons in a polysensory area in superior temporal sulcus of the macaque. J Neurophysiol 1981, 46:369-384.

23. Cusick CG: The superior temporal polysensory region in monkeys. Cerebral Cortex: Extrastriate Cortex in Primates 1997, 12:435-468.

24. Beauchamp MS, Lee KE, Argall BD, Martin A: Integration of auditory and visual information about objects in superior temporal sulcus. Neuron 2004, 41:809-823.

25. Noesselt T, Rieger JW, Schoenfeld MA, Kanowski M, Hinrichs H, Heinze HJ, Driver J: Audiovisual temporal correspondence modulates human multisensory superior temporal sulcus plus primary sensory cortices. J Neurosci 2007, 27:11431-11441.

26. Calvert GA: Crossmodal processing in the human brain: Insights from functional neuroimaging studies. Cereb Cortex 2001, 11:1110-1123.

27. Van Atteveldt NM, Formisano E, Blomert L, Goebel R: The effect of temporal asynchrony on the multisensory integration of letters and speech sounds. Cereb Cortex 2007, 17:962-974.

28. Bischoff M, Walter B, Blecker CR, Morgen K, Vaitl D, Sammer G: Utilizing the ventriloquism-effect to investigate audio-visual binding. Neuropsychologia 2007, 45:578-586.

29. Dhamala M, Assisi CG, Jirsa VK, Steinberg FL: Scott Kelso JA: Multisensory integration for timing engages different brain networks. Neuroimage 2007, 34:764-773.

30. Reale RA, Calvert GA, Thesen T, Jenison RL, Kawasaki H, Oya H, Howard MA, Brugge JF: Auditory-visual processing represented in the human superior temporal gyrus. Neuroscience 2007, 145:162-184.

31. Szycik GR, Jansma H, Munte TF: Audiovisual integration during speech comprehension: an fMRI study comparing ROI-based and whole brain analyses. Hum Brain Mapp 2009, 30:1990-1999.

32. Sekiyama K, Kanno I, Miura S, Sugita Y: Auditory-visual speech perception examined by fMRI and PET. Neurosci Res 2003, 47:277-287.

33. Calvert GA, Campbell R, Brammer MJ: Evidence from functional magnetic resonance imaging of crossmodal binding in the human heteromodal cortex. Curr Biol 2000, 10:649-657. 
34. Callan DE, Callan AM, Kroos C, Vatikiotis-Bateson E: Multimodal contribution to speech perception revealed by independent component analysis: A single-sweep EEG case study. Cogn Brain Res 2001, 10:349-353.

35. Möötönen $R$, Schürmann $M$, Sams M: Time course of multisensory interactions during audiovisual speech perception in humans: A magnetoencephalographic study. Neurosci Lett 2004, 363:112-115.

36. Jeannerod M: The representing brain: Neural correlates of motor intention and imagery. Behav Brain Sci 1994, 17:187-245.

37. Binkofski F, Buccino G, Stephan KM, Rizzolatti G, Seitz RJ, Freund HJ: A parietopremotor network for object manipulation: Evidence from neuroimaging. Exp Brain Res 1999, 128:210-213.

38. Buccino G, Binkofski F, Fink GR, Fadiga L, Fogassi L, Gallese V, Seitz RJ, Zilles K, Rizzolatti G, Freund HJ: Action observation activates premotor and parietal areas in a somatotopic manner: An fMRI study. Eur J Neurosci 2001, 13:400-404.

39. Fadiga L, Fogassi L, Pavesi G, Rizzolatti G: Motor facilitation during action observation: A magnetic stimulation study. J Neurophysiol 1995, 73:2608-2611.

40. Gallese V, Fadiga L, Fogassi L, Rizzolatti G: Action recognition in the premotor cortex. Brain 1996, 119:593-609.

41. Gallese V, Fogassi L, Fadiga L, Rizzolatti G: Action representation and the inferior parietal lobule. Common Mechanisms in Perception and Action: Attention Perform 2002, 19:334-355.

42. Gazzola V, Rizzolatti G, Wicker B, Keysers C: The anthropomorphic brain: The mirror neuron system responds to human and robotic actions. Neuroimage 2007, 35:1674-1684.

43. Rizzolatti G, Fadiga L: Grasping objects and grasping action meanings: The dual role of monkey rostroventral premotor cortex (area F5). In Novartis Foundation Symposium. Edited by Glickstein M. Chichester, UK: John Wiley\& Sons; 1998:81-103.

44. Di Pellegrino G, Fadiga L, Fogassi L, Gallese V, Rizzolatti G: Understanding motor events: A neurophysiological study. Exp Brain Res 1992, 91:176-180.

45. lacoboni M, Koski LM, Brass M, Bekkering H, Woods RP, Dubeau MC, Mazziotta JC, Rizzolatti G: Reafferent copies of imitated actions in the right superior temporal cortex. Proc Natl Acad Sci USA 2001, 98:13995-13999.

46. lacoboni M: Understanding others: Imitation, language, empathy. In Perspectives on Imitation: From Mirror Neurons to Memes. Edited by Hurley S, Chater N. Cambridge: MIT Press; 2004:32-45.

47. Grafton ST, Arbib MA, Fadiga L, Rizzolatti G: Localization of grasp representations in humans by positron emission tomography. 2 . Observation compared with imagination. Exp Brain Res 1996, 112:103-111.

48. Grafton ST, Hazeltine E, Ivry RB: Motor sequence learning with the nondominant left hand: A PET functional imaging study. Exp Brain Res 2002, 146:369-378.

49. Decety J, Grezes J, Costes N, Perani D, Jeannerod M, Procyk E, Grassi F, Fazio F: Brain activity during observation of actions. Influence of action content and subject's strategy. Brain 1997, 120:1763-1777.

50. Decety J, Grezes J: Neural mechanisms subserving the perception of human actions. Trends Cogn Sci 1999, 3:172-178.

51. Perani D, Fazio F, Borghese NA, Tettamanti M, Ferrari S, Decety J, Gilardi MC: Different brain correlates for watching real and virtual hand actions. Neuroimage 2001, 14:749-758.

52. Tai YF, Scherfler C, Brooks DJ, Sawamoto N, Castiello U: The Human Premotor Cortex Is 'Mirror' only for Biological Actions. Curr Biol 2004, 14:117-120.

53. Stevens JA, Fonlupt $P$, Shiffrar M, Decety J: New aspects of motion perception: Selective neural encoding of apparent human movements. Neuroreport 2000, 11:109-115.

54. Buccino G, Lui F, Canessa N, Patteri I, Lagravinese G, Benuzzi F, Porro CA Rizzolatti G: Neural Circuits Involved in the Recognition of Actions Performed by Nonconspecifics: An fMRI Study. J Cogn Neurosci 2004, 16:114-126.

55. Puce A, Perrett D: Electrophysiology and brain imaging of biological motion. Phil Trans Roy Soc B: Biol Sci 2003, 358:435-445.

56. Allison T, Puce A, McCarthy G: Social perception from visual cues: Role of the STS region. Trends Cogn Sci 2000, 4:267-278.

57. Saxe R: Uniquely human social cognition. Curr Opin Neurobiol 2006 $16: 235-239$.

58. Saxe R, Kanwisher N: People thinking about thinking people: The role of the temporo-parietal junction in "theory of mind". Neuroimage 2003 19:1835-1842
59. Zilbovicius M, Meresse I, Chabane N, Brunelle F, Samson Y, Boddaert N: Autism, the superior temporal sulcus and social perception. Trends Neurosci 2006, 29:359-366.

60. Amedi A, Von Kriegstein K, Van Atteveldt NM, Beauchamp MS, Naumer MJ: Functional imaging of human crossmodal identification and object recognition. Exp Brain Res 2005, 166:559-571.

61. Beauchamp MS: See me, hear me, touch me: Multisensory integration in lateral occipital-temporal cortex. Curr Opin Neurobiol 2005, 15:145-153.

62. Driver J, Noesselt T: Multisensory Interplay Reveals Crossmodal Influences on 'Sensory-Specific' Brain Regions, Neural Responses, and Judgments. Neuron 2008, 57:11-23.

63. Hein G, Knight RT: Superior Temporal Sulcus-It's My Area: Or Is It? J Cogn Neurosci 2008, 20:1-12.

64. Agostini T, Righi G, Galmonte A, Bruno P: The relevance of auditory information in optimizing hammer throwers performance. In Biomechanics and sports. Edited by Pascolo PB. Wien: Springer; 2004:67-74.

65. Schaffert N, Mattes K, Barrass S, Effenberg AO: Exploring function and aesthetics in sonifications for elite sports. In Proceedings of the 2 nd International Conference on Music Communication Science (ICOMCS2). Edited by Stevens C, Schubert E, Kruithof B, Buckley K, Fazio S. Sydney: HCSNet; 2009:83-86.

66. Schaffert N, Mattes K, Effenberg AO: A Sound Design for Acoustic Feedback in Elite Sports. In Auditory Display. CMMR/ICAD 2009, Lecture Notes in Computer Science (LNCS) Vol. 5954. Edited by Ystad S. Berlin: Springer; 2010:143-165.

67. Schaffert N, Mattes K, Effenberg AO: Die Bootsbeschleunigung als akustisches Feedback im Rennrudern. In Bewegung und Leistung? Sport, Gesundheit \& Alter. Schriften der Deutschen Vereinigung für Sportwissenschaft. Bd. 204. Edited by Mattes K, Wollesen B. Hamburg: Feldhaus; 2010:28.

68. Hummel J, Hermann T, Frauenberger C, Stockman T: Interactive sonification of German wheel sports movement. In Proceedings of ISon 2010, 3rd Interactive Sonification Workshop, KTH, Stockholm, Sweden, April 7, 2010. Stockholm:: 2007:17-22

69. Kleiman-Weiner $M$, Berger J: The sound of one arm swinging: a model for multidimensional auditory display of physical motion. In Proceedings of the 12th International Conference on Auditory Display. London, UK: 2006:278-280.

70. Bangert $M$, Altenmüller EO: Mapping perception to action in piano practice: a longitudinal DC-EEG study. BMC Neuroscience 2003, 4:26.

\section{doi:10.1186/1471-2202-14-32}

Cite this article as: Schmitz et al:: Observation of sonified movements engages a basal ganglia frontocortical network. BMC Neuroscience 2013 $14: 32$.

\section{Submit your next manuscript to BioMed Central and take full advantage of:}

- Convenient online submission

- Thorough peer review

- No space constraints or color figure charges

- Immediate publication on acceptance

- Inclusion in PubMed, CAS, Scopus and Google Scholar

- Research which is freely available for redistribution 